\title{
A HETEROGENEOUS MODEL FOR HEAT TRANSFER IN PACKED BEDS
}

\author{
R. J. WIJNGAARDEN ${ }^{\dagger}$ and $K$. R. WESTERTER $P^{\ddagger}$ \\ Chemical Reaction Engineering Laboratories, Department of Chemical Engineering, Twente University, \\ PO Box 217, 7500 AE Enschede, The Netherlands
}

(Received 8 July 1991; accepted for publication 16 July 1992)

\begin{abstract}
Ahstract-If transient heat transfer occurs in a packed bed or a reaction is carried out on the pellets, the heterogeneity of the bed is essential because of the heat flow between pellets and gas. Global heat parameters for the packed bed, such as $\lambda_{\text {efr }}$ and $\alpha_{w \text { s }}$ are usually derived from homogeneous models. Therefore, one must choose whether to attribute the values of $\lambda_{e f f}$ and $\alpha_{w}$ to the gas phase, solid phase or to both the phases. To describe changing radial and axial temperature profiles in a packed bed we assumed the heat transport in the solid and gas phase to occur in series by three mechanisms: heat transfer from the solid to the gas, described by the pellet heat transfer coefficient, $\alpha_{p}$; heat transfer through the gas to the outer wall, described by the effective radial heat conductivity, $\lambda_{\text {eff; }}$ and heat transfer at the wall through the gas, given by the coefficient $\alpha_{w}$. The values of $\lambda_{\text {eff }}, \alpha_{w}$ and $\alpha_{p}$ were determined by fitting the measured temperature profiles with a dynamic heterogeneous model. Two different-diameter beds, packed with industrial, ring-shaped pellets, were employed. The superficial gas velocity, based on the empty tube, was varied up to $3 \mathrm{~m} / \mathrm{s}$. Air was used as the gas. The values obtained for $\lambda_{e f f}, \alpha_{w}$ and $\alpha_{p}$ are in good agreement with the values reported in the literature. Also, the agreement between the measured temperature profiles and the profiles obtained by fitting is very good. This confirms that the series model is a reasonable assumption and is close to physical reality. Therefore, it is concluded that, if packed-bed heterogeneity is significant, the above series model can be used to describe the temperature profiles.
\end{abstract}

\section{INTRODUCTION}

Many studies have been conducted on the radial effective heat conductivity, $\lambda_{\text {eff }}$, and the heat transfer coefficient at the wall, $\alpha_{w}$ in packed beds [see e.g. Zehner (1973). Hennecke and Schlünder (1973), Zehner and Schlünder (1973), Lerou and Froment (1978), Bauer (1977), Dixon and Cresswell (1979), Hofmann (1979) and Wellauer et al. (1982)]. Reviews in this field have been given by Westerterp et al. (1987) and Tsotsas and Martin (1987). All the authors use homogeneous models to describe the packed bed, i.e. they assume that the pellets and the gas have the same properties. The heterogeneous character of the packed bed is lumped into the values of $\lambda_{\text {ett }}$ and $\alpha_{w}$ of the pseudo-homogeneous models.

Also for the heat transfer coefficient between the pellet and the gas, many results have been reported [see e.g. Ranz (1952)]. More recently, Gnielinski (1980, 1982) gave correlations which can be used for high particle Reynolds numbers and which cover a wide range of data presented in the literature. For low particle Reynolds numbers the Nusselt number decreases rapidly to nil; in this region the correlations of Ranz (1952) and Gnielinski $(1980,1982)$ fail to give a good description. Kunif and Suzuki (1967) try to explain that in packed beds Nusselt numbers drop well below the limiting value of two, which holds for a single sphere in a gas stream at very low fluid

\footnotetext{
'Present address: Koninklijke/Shell-Laboratorium, Amsterdam, PO Box 3003, 1003 AA Amsterdam, The Netherlands.

'Author to whom correspondence should be addressed.
}

velocities. Attempts to explain this phenomenon have also been made by Gunn and de Souza (1974), Nelson and Galloway (1975) and Martin (1978).

Particle heat transfer coefficients are often obtained from dynamic measurements in packed beds. Essential for these measurements is that the packed bed is heterogeneous in nature so that the gas and solid phase have different temperatures.

For transient temperature profiles in packed beds, on the one hand, the heterogeneity of the packed bed is essential to describe the pellet-scale heat transfer between the gas and the solid phase; on the other hand, the heterogeneity cannot be accounted for since we have only values for the bed-scale properties, $\lambda_{\text {eff }}$ and $\alpha_{w}$, arising from homogeneous models. Rather than accounting for radial heat transport in the bed by taking heat transport in parallel via the gas and solid phase - in which case we would have two values for $\lambda_{\text {eff }}$ and two values for $\alpha_{w,}$ for the gas and for the solid phase each-we assumed that heat transport takes place via the gas and the solid phase in series. That is, heat from the solid phase is transferred to the gas and back again to adjacent particles, which is described by $\alpha_{p}$, and in the gas phase to the wall, which is described by $\lambda_{\text {eff }}$ and $\alpha_{w^{-}}$Cresswell (1986) mentions a combination of the series and parallel model.

\section{EXPEKIMIENTAL}

A packed bed was brought entirely to an elevated, constant temperature. As of the moment zero the gas flow was switched to cold air. The wall temperature was kept equal to the initial temperature of the 
packed bed. The experiment was terminated as soon as a stationary profile was reached. Thermocouples had been shoved in the packed bed via small guiding tubes placed vertically in the packed bed and at varying radial distances. In this way, temperatures could be measured as a function of axial position, radial position and time. The obtained values are the gas temperatures, not the pellet temperatures.

We used two packed beds: a large one with a diameter of $50 \mathrm{~cm}$ and a height of $40 \mathrm{~cm}$ and a small one with a diameter of $22 \mathrm{~cm}$ and a height of $20 \mathrm{~cm}$ (see Fig. 1). In the large packed bed superficial gas velocities up to $15 \mathrm{~cm} / \mathrm{s}$ could be reached, in the small bed up to $3 \mathrm{~m} / \mathrm{s}$.

In the centre axis of the large packed bed a smalldiameter pipe with an outer diameter of $20 \mathrm{~mm}$ was placed (see Fig. 1). Through this pipe cold water flowed, thus allowing us to examine the effect of the curvature of the wall on $\alpha_{w}$. We obtained two values for $\alpha_{w}$ for the inner pipe and the outer wall, respectively. In the small packed bed only the $\alpha_{w}$ value for the outer wall was obtained.

The pellet material was an industrial ring-shaped cataslyst (see Fig. 2). In all the experiments air was used as the gas.

\section{THEORETICAL}

It is assumed that accumulation of heat in the gas phase can be neglected. Furthermore, axial dispersion of heat in both the solid and the gas phase is neglected, as well as heat conduction within the particles. The last assumptions have been justified by Wijngaarden (1988). Finally, radial porosity, velocity and heat conductivity profiles have not been incorporated in the model. This still gives an accurate enough description because the ratio of the packed bed to particle diameter is always larger than 35 . The high gas velocities locally near the packed-bed wall are lumped in a wall heat transfer coefficient, $\alpha_{w}$. It has been argued that inclusion of the radial velocity profile near the wall gives a somewhat more accurate description [see Vortmeyer and Haidegger (1991)]. However, because of the small wall effects in the set-ups under consideration, it was decided to work with the concept of an $\alpha_{w}$ to avoid extreme complexity of the models.

Given the above assumptions, a heat balance for an infinitesimally small ring in the packed bed yields

$$
\begin{gathered}
\frac{\partial \theta_{g}}{\partial \omega}-\frac{1}{P e^{\ominus}} \frac{1}{\rho} \frac{\partial}{\partial \rho}\left(\rho \frac{\partial \theta_{g}}{\partial \rho}\right)+S t^{\ominus}\left(\theta_{g}-\theta_{k}\right)=0 \\
S t^{\ominus}\left(\theta_{g}-\theta_{k}\right)-\frac{\partial \theta_{k}}{\partial \tau}=0
\end{gathered}
$$

subject to the initial conditions

$$
\begin{aligned}
\omega & =0 \Rightarrow \theta_{a}=\theta_{\text {in }} \\
\tau & =0 \Rightarrow \theta_{k}=\theta_{b}
\end{aligned}
$$

and the boundary conditions

$$
\begin{gathered}
\rho=1 \Rightarrow \frac{\partial \theta_{g}}{\partial \rho}=B i_{c}^{\ominus} \theta_{\theta} \\
\rho=1 \Rightarrow \frac{\partial \theta_{g}}{\partial \rho}=B i_{h}^{\ominus}\left(1-\theta_{g}\right) .
\end{gathered}
$$

The definition of the dimensionless numbers can be found in the notation. Equations $(1)-(6)$ can be solved to yield the formulae given in the Appendix.

With the formulae given in the appendix the dimensionless temperatures $\theta_{\theta}$ and $\theta_{k}$ can be calculated as a function of $\omega, \rho$ and $\tau$ for any chosen values of $P e^{\ominus}$, $B i_{h}^{\ominus}, B i_{c}^{\ominus}$ and $S t^{\ominus}$. This is illustrated in Fig. 3. In this figure $\theta_{g}$ and $\theta_{k}-\theta_{g}$ are plotted vs $\omega$ and $\rho$ for several values for $\tau$. In the caption of the figure the values which were chosen for $P e^{\ominus}, B i_{h}^{\ominus}, B i_{c}^{\ominus}$ and $S t^{\ominus}$ can be found and also the values of $\theta_{\text {in }}$ and $\theta_{b}$, zero and one,

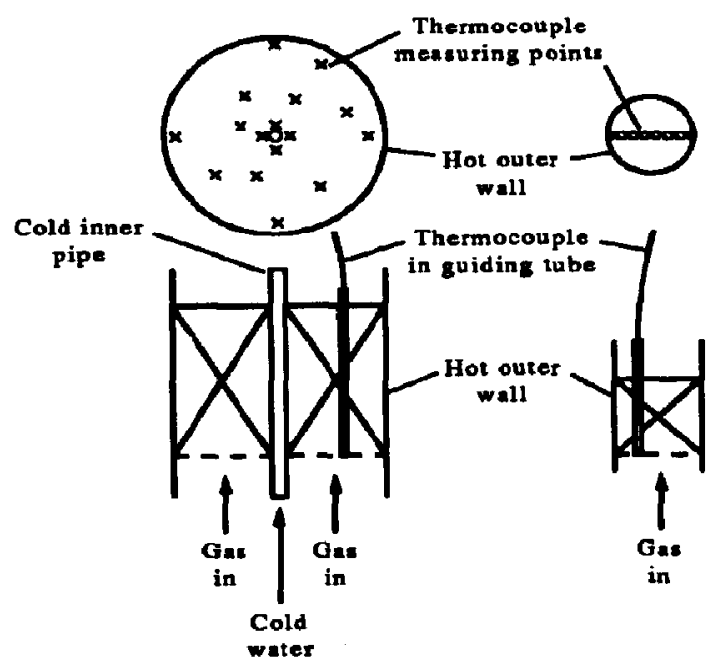

Fig. 1. The set-ups employed in this investigation.

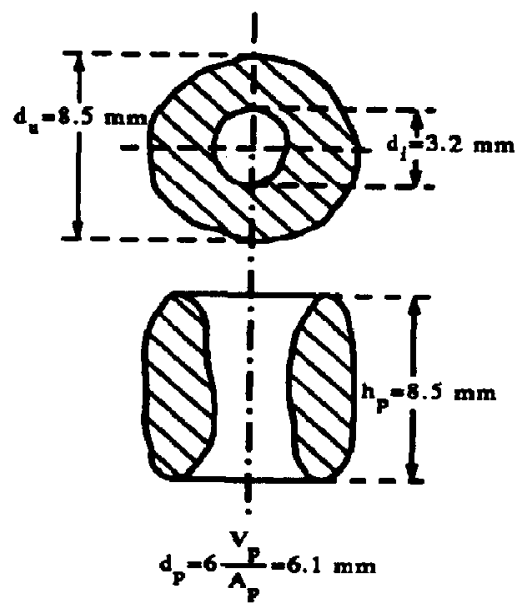

Fig. 2. The geometry of the pellets employed. 

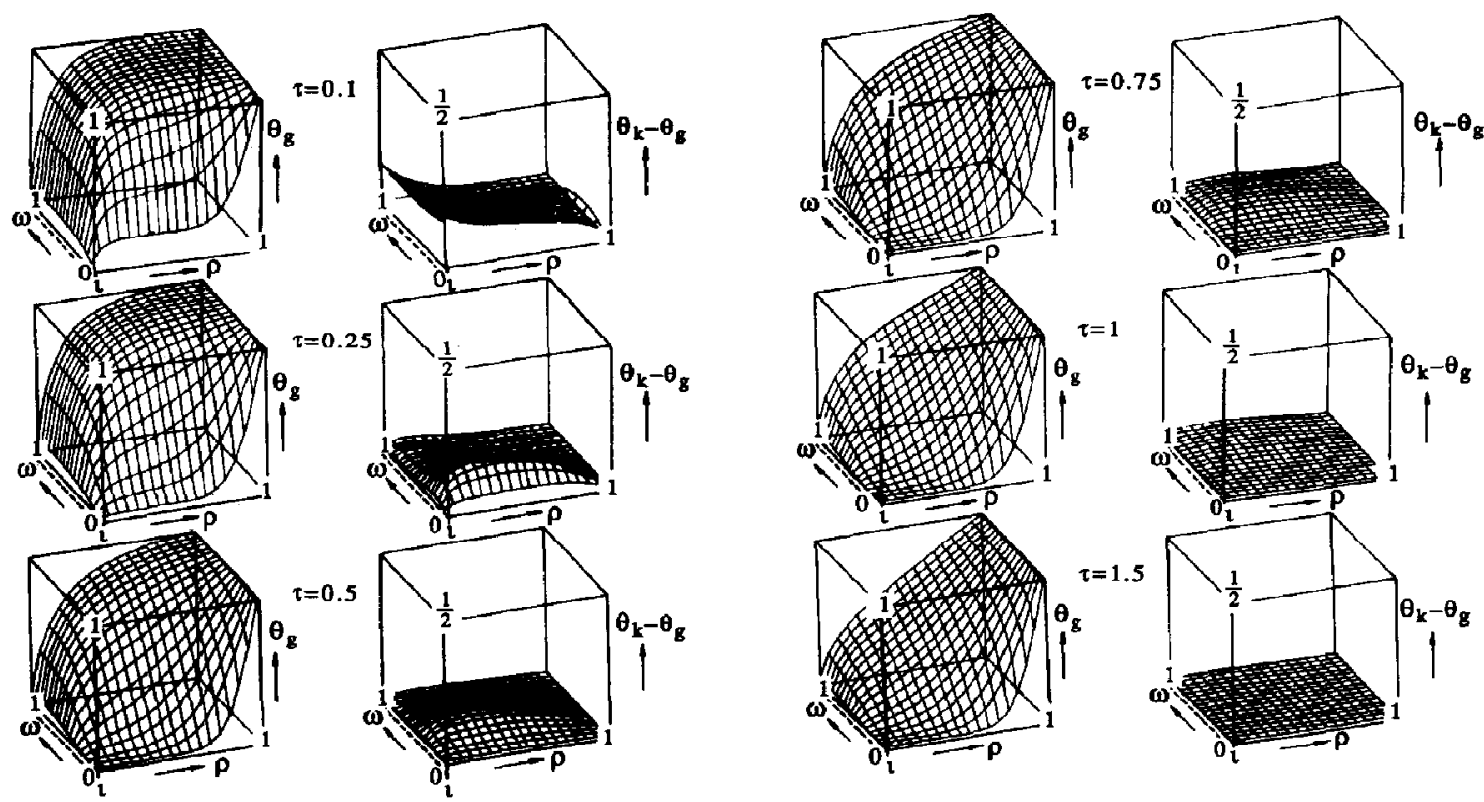

Fig. 3. The gas temperature and the temperature difference between solid and gas in the bed at different moments of time. The figures were obtained from the formulae given in the appendix. Further, $P e^{\ominus}=5$, $S t^{\ominus}=20, B i_{c}^{\Theta}=B i_{h}^{\ominus}=100$ and $\theta_{i n}=0, \theta_{b}=1$. For symbols see the notation.

respectively. So, the inlet temperature of the gas equals the temperature of the cold inner pipe and the initial temperature of the bed equals the temperature of the hot outer wall. Notice that from the moment of time zero a temperature front travels through the packed bed in the axial direction. As it proceeds, the slope in its point of inflection declines, i.e. the front becomes less steep. For a dimensionless time $\tau$ roughly equalling unity, the front becomes steady and a stationary profile is established throughout. The difference in temperature between the pellets and the gas is the highest in the point of inflection of the temperature front, and with time it decreases. Eventually, in the steady state it has vanished completely. At $\tau=1.5$ the difference $\theta_{k}-\theta_{\theta}$ has become smaller than 0.01 .

The dimensionless groups $P e^{\ominus}, B i_{h}^{\ominus}, B i_{c}^{\ominus}$ and $S t^{\ominus}$ can be regarded as compounded parameters. They consist of products of morc fundamental groups:

$$
\begin{aligned}
P e^{\ominus} & =\frac{P e N_{D}}{4 \Gamma \Lambda} \\
B i_{h}^{\ominus} & =\frac{N_{D} B i_{h}}{2} \\
B i_{c}^{\ominus} & =\frac{N_{D} B i_{c}}{2} \\
S t^{\ominus} & =\frac{(1-\varepsilon) N_{D} \Lambda N u}{6 P e} .
\end{aligned}
$$

The measured gas temperature profiles were fitted with a procedure similar to the direct search method.
Table 1. Values of the fundamental geometrical groups of the packed beds used for the measurements

\begin{tabular}{lcc}
\hline & $\begin{array}{l}\text { Large } \\
\text { set-up }\end{array}$ & $\begin{array}{c}\text { Smal1 } \\
\text { set-up }\end{array}$ \\
\hline$N_{D}$ & $\mathbf{8 3}$ & 36 \\
$\Lambda$ & $\mathbf{0 . 8 0}$ & 0.91 \\
$t$ & $\mathbf{0 . 0 4 0}$ & 0 \\
$\epsilon$ & $\mathbf{0 . 4 8}$ & 0.48 \\
\hline
\end{tabular}

The truncation error of the method was smaller than $1 \%$. As a result of this procedure we obtain best-fit values for the compounded parameters $P e^{\ominus}, B i_{h}^{\ominus}$, $B i_{c}^{\Theta}$ and $S t^{\ominus}$. This was done for several gas velocities. The superficial gas velocity, $v_{0}$, was measured for every experiment; from this the Peclet number, $P e=v_{0} d_{p} / a_{g}$, was obtained with $d_{p}=6.1 \times 10^{-3} \mathrm{~m}$ and for air $a_{g}=4.8 \times 10^{-5} \mathrm{~m}^{2} / \mathrm{s}$. For both packed beds the porosity $\varepsilon$ was measured by determining the volume of the bed, the total mass of the pellets in the bed and the pellet density. The pellet density was found by measuring the mass of a number of pellets and the corresponding upward force in mercury. The geometrical fundamental groups $N_{D}, \Lambda$ and $i$ were calculated. The results are summarized in Table 1 . Then according to eqs (7)-(10), from the parameters $P e^{\ominus}, B i_{h}^{\ominus}, B i_{c}^{\ominus}$ and $S t^{\ominus}$, the groups $\Gamma, B i_{k} B i_{c}$ and $N u$ were obtained and after that plotted as a function of $P e$ for both the packed beds. 


\section{RESIITS AND DISCITSSION}

\section{Temperature profiles}

The small packed bed and the large packed bed behave differently. The heterogeneous character of a packed bed on a pellet scale can induce scatter in the measured temperature profiles. The randomness of the packing is another source for scatter; however, here we will discuss the role of the packed bed heterogeneous character. A point in the bed is situated either in the gas phase or in the solid phase. Since, in general, the gas and the solid phase will not have the same heat conductivities, the temperature gradient in both the phases will have to be different to arrive at the same heat flux. Hence, the radial temperature profiles usually will not be differentiable on the boundary between a pellet and its surrounding gas. If external heat transfer between a pellet and its surrounding gas plays a role, there will even be a temperature jump between the pellet and the gas. Then the temperature profile will also be discontinuous on the boundary between the pellet and the gas.

Imagine a packed bed heated at the wall through which a cold gas flows. Through the bed we draw a line which intersects with the centre axis of the bed. If we plot the temperature as a function of the distance along this line $(r)$, a plot as illustrated in Fig. 4 is obtained (the results in Fig. 4 have not becn mcasured, but merely serve to illustrate a point). Instead of a smooth parabolically shaped profile we obtain a profile full of jumps and bends. This is confirmed by Cresswell (1986), who found a $10^{\circ} \mathrm{C}$ spread for a total gradient of $40^{\circ} \mathrm{C}$. An example of temperature profiles measured by us in the small packed bed is given in Fig. 5. Between the measurements the packed bed was fluidized for a few seconds and allowed to settle again, so that a new random packing was obtained. The profiles shown in Fig. 5 are the steady-state profiles, measured half-way up in the packed bed. The thin lines represent individual temperature profiles; the bold line gives the average profile. Notice that although the individual profiles show a considerable amount of scatter, the average profile is rather smooth; also, this is confirmed by Cresswell (1986). The deviation between these average profiles and the profiles obtained by filting was smaller than $1 \% \mathrm{C}$ per thermocouple.

For the large packed bed the individual profiles were satisfactory. Because of the large amount of particles between the measuring probes, the amount of scatter in the individual temperature profiles was so small, that the difference between the profile obtained by fitting and an individually measured profile was already smaller than $1^{\circ} \mathrm{C}$ per thermocouple. This is illustrated in Fig. 6, where a stationary radial temperature profile is plotted for the large bed. The dots are the gas temperatures measured in the packed bed; the line gives the profile obtained by fitting.

For the small packed-bed values of $\Gamma, B i$ and $\mathrm{Nu}$ were obtained by fitting the average of 20 temperature profiles, where between the individual profile measurements the bed was fluidized. For the large bed only one profile measurement was done at one gas velocity without repeating after fluidization.

\section{Effective radial heat conductivity}

In Fig. 7 the results found for $\lambda_{\text {efr }}$ are presented as $\Gamma$ plotted vs $\mathrm{Pe}$. Two different sets of points are given: the small points between $0<P e<40$ for the large packed bed and the large dots for the small bed. Also, the correlation given by Zehner and Schlünder (1973)

- - - Ideal, parabola shaped profile

Actual, measured profile

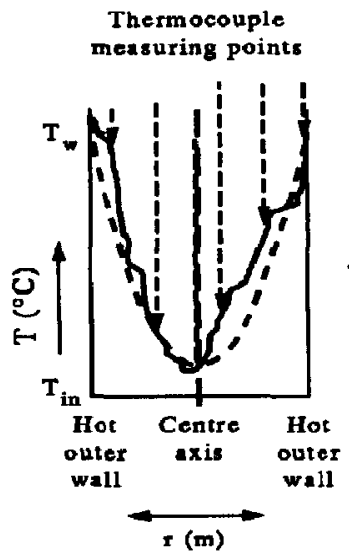

(a)

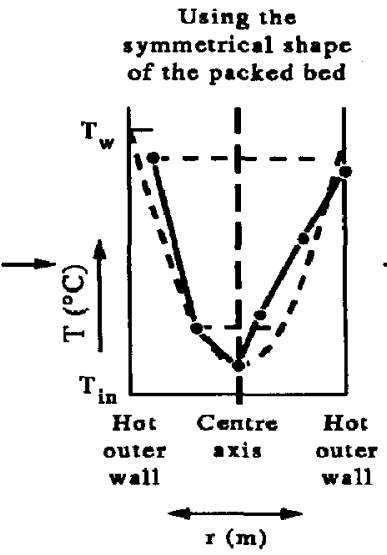

(b)
Prafile obtained
after projection

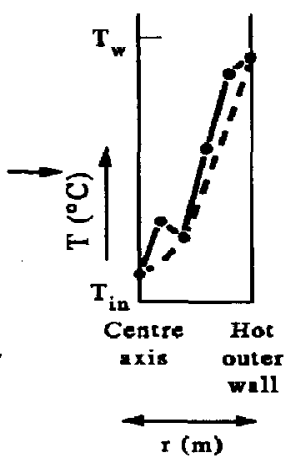

(c)

Fig. 4. The heterogeneity of the packed bed on a pellet scale will induce scatter in the measured temperature profiles. In situation (a) an example of a measured temperature profile is given, in (b) these temperatures are projected on the right side of the cylinder and in (c) the results after projection are shown. 


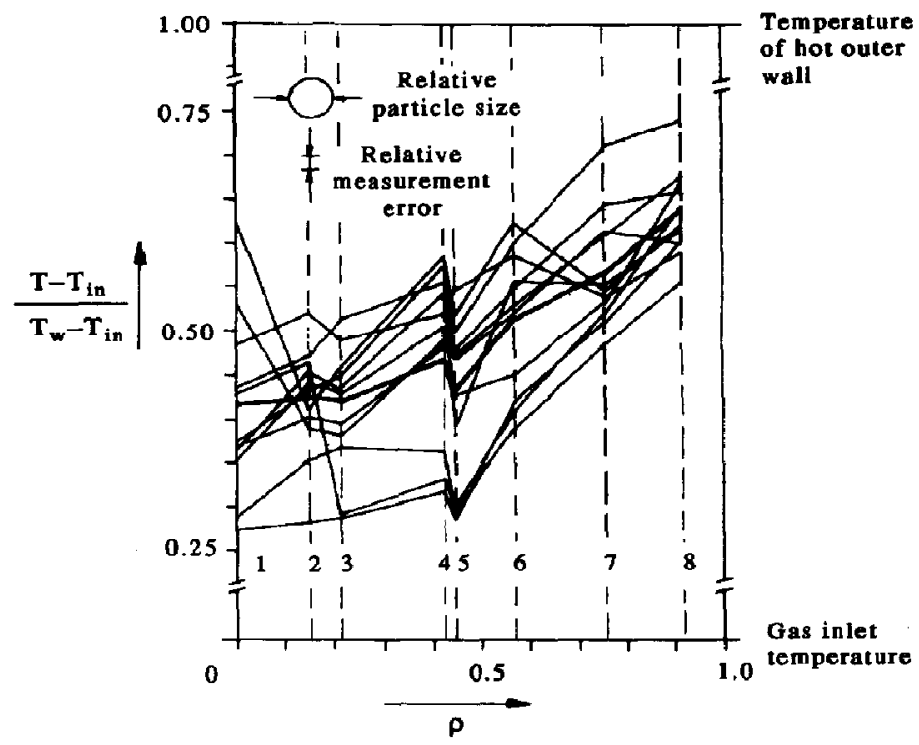

Fig. 5. Some radial temperature profiles which were measured in the small packed bed. The bold line gives the average of the individual profiles. The relative measurement error refers to the relative error in the thermocouple.

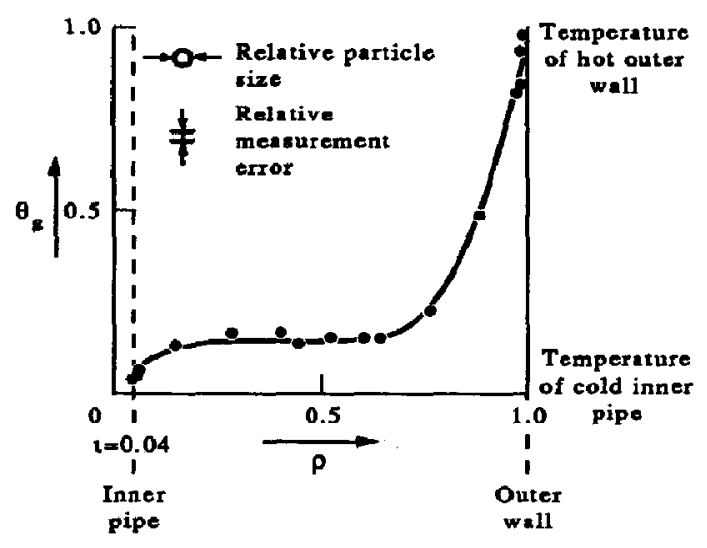

Fig. 6. A radial temperature profile which was measured in the large packed bed. The line represents the best fit obtained with the model given in the appendix.

is shown in Fig. 7 for both the beds. These are the two straight lines drawn in the figure. From these lines we can conclude that the effect of the number of particles on a diameter should be negligible.

The values of $\Gamma$ as measured in the large packed bed all lie in the same order as the values calculated with the correlation. For the small packed bed the spread amounts to a factor of three. This is explained by the bed-scale statistical behaviour [see Wijngaarden and Westerterp (1992)].

\section{Heat transfer coefficient at the wall}

In Fig. 8 the results obtained for $\alpha_{w}$ are presented, where $B i$ is plotted vs $P e$. Three sets of points can be distinguished:

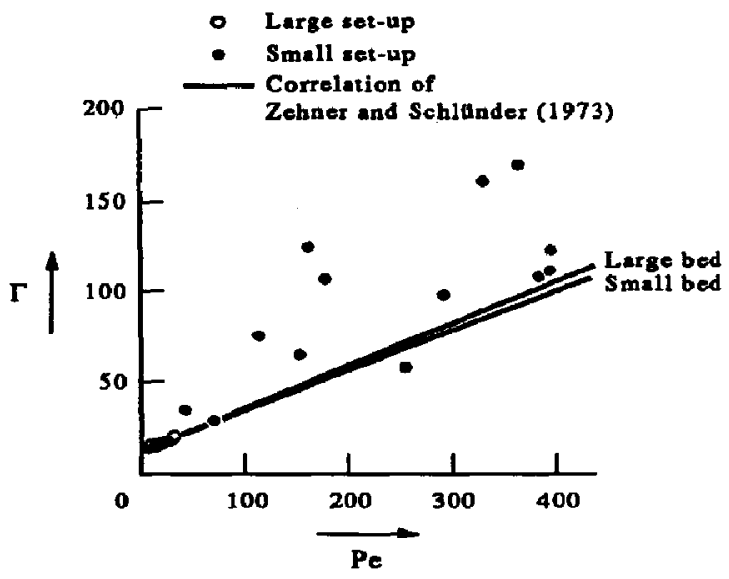

Fig. 7, Effective radial heat conductivity vs velocity.

(1) $\alpha_{w}$ for the outer wall in the large set-up,

(2) $\alpha_{w}$ for the small inner pipe in the large set-up,

(3) $\alpha_{w}$ for the outer wall in the small set-up.

Also, the correlation of Dixon and Cresswell (1979) is shown in Fig. 8. According to this correlation,

$$
B i=2.8 P e^{-0.25}, \quad 30<P e<4000 \text {. }
$$

Again, the values measured by us and calculated with the correlation lie in the same order. Two sets out of three show a larger amount of scatter: the $\alpha_{w}$ at the outer wall of the large set-up and the $\alpha_{w}$ in the small set-up. This can be explained as follows. For the large set-up the temperature profile is very steep near the outer wall, whereas the slope is moderate near the 


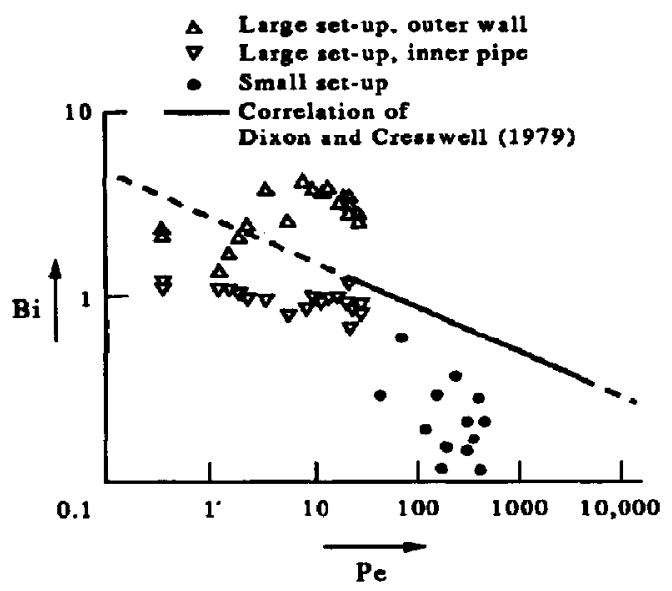

Fig. 8. The ratio of the heat transfer coefficient at the wall and the effective radial heat conductivity, $B i-B i_{c}$ and $B i_{h}$, respectively, for the large set-up_vs the velocity $P e$.

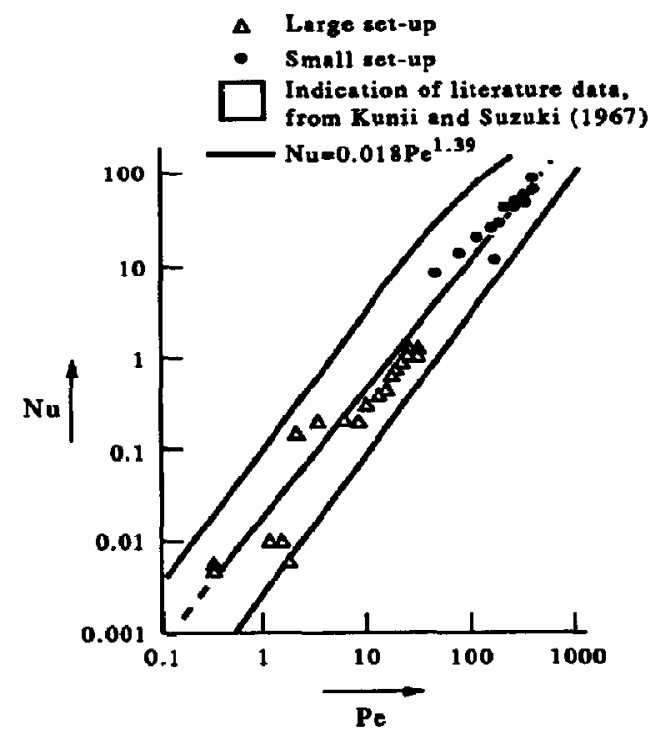

Fig. 9. The pellet heat transfer coefficient $\mathrm{Nu}$ vs $\mathrm{Pe}$.

inner tube (see e.g. Fig. 6). As a result of the difference in steepness, a slight error in the radial position of the thermocouple will induce a large error in the temperature near the outer wall; near the inner tube the induced error will be small. This measurement error explains why for the large set-up the scatter is much larger for $\alpha_{w}$ at the outer wall than for $\alpha_{w}$ at the inner tube. For the small set-up the scatter in temperature induces scatter in the values of $\alpha_{w}$. We see that the results seem to confirm the type of dependence which was suggested by Dixon and Cresswell (1979).

Heat transfer coefficient between the pellet and the gas

In Fig. 9 the results obtained for $\alpha_{p}$ are presented, where $\mathrm{Nu}$ is plotted vs $\mathrm{Pe}$. Two sets of points can be distinguished: one set for the large set-up and one for the small set-up. General correlations for $\mathrm{Nu}$ vs $\mathrm{Pe}$, covering the whole range of Peclet values, are not given in the literature. To compare our data we indicated the data given in the survey of Kunii and Suzuki (1967) in Fig. 9. Notice that the agreement between our results and the results presented in the literature is very satisfactory: all points lie within the indicated area. The results for the large and small set-up are also in good agreement with cach other. A correlation for $N u$ for the pellet geometry used by us yields

$$
N u=0.018 P e^{1.39}, \quad 0.4<P e<400 .
$$

This correlation, which is the best fit obtained for the Peclet range for which the measurements were carried out, is represented by the line in Fig. 9. The average deviation between the measured values and those calculated with this correlation is $9 \%$. We want to emphasize that to arrive at this correlation two setups were used and the pellet geometry was that of a Raschig ring; further, only air was used as the gas.

\section{CONCLUSIONS}

To a certain extent the choice of a series model is a rather arbitrary one: all heat transport properties in a packed bed are lumped parameters, and model deviations are lumped in the values of these properties. Since no model matches reality completely, we will always lump the relevant physical processes in one of the values of the heat transport properties. If a model is closer to the physical reality, the correlations obtained are better suited for extrapolation to situations where other physical phenomena play a role. This urges for the choice of a model which is as close to physical reality as possible.

There are several reasons why we believe the series model is close to physical reality. The static contribution of the effective heat conductivity of the bed depends un the heat conductivity of the solid phase. This is in agreement with the series model: heat is transported from one pellet to another via the gas phase, although also heat is transferred through the pellet itself. Our series model states that heat transport via the contact spot is negligible. This is in agreement with the results of Bauer (1977), who shows that the contact-spot area between pellets in a packed bed is so small that the contribution of this contact spot to the static contribution of the effective heat conductivity of the bed is virtually negligible.

It has been argued that parallel heat transport cannot be neglected because the stagnant gas interstices near the pellet contact points must be included as part of the solid phase. We do not see any reason to include these interstices as part of the solid phase, since they simply consist of gas. This region will contribute to the overall value of the heat transfer coefficient of a single particle in a bed to its surrounding gas. 
Finally, using a series model, we found that the values of $\alpha_{p}, \lambda_{\text {eff }}$ and $\alpha_{w}$ obtained by fitting the measured temperature profiles are in good agreement with the values reported in the literature. Also, the agreement between the measured temperature profiles and the profiles as calculated is very good; we found that the average deviations were smaller than $1^{\circ} \mathrm{C}$ per thermocouple for a total temperature gradient in the bed of $50^{\circ} \mathrm{C}$. In other words, we could predict the measured temperature profiles very well, assuming a series model.

Therefore, we believe that the series model is close to physical reality and recommend its use for packedbed heterogeneous models.

Acknowledgements - The investigations have been and are supported, in part, by the Netherlands' Foundation for Chemical Research SON, with financial aid from the Netherlands' Technology Foundation STW.

\section{NOTATION}

$a_{g}$ thermal diffusivity of the gas, $\mathrm{m}^{2} / \mathrm{s}$

$A_{p}$ external surface area of the pellet, $\mathrm{m}^{2}$

$B i$ Biot number based on particle diameter $\left(=\alpha_{w} d_{p} / \lambda_{\text {eff }}\right)$, dimensionless

$B i_{c} \quad$ Biot number based on particle diameter for the hot outer wall in the large set-up ( $=\alpha_{w, c} d_{p} / \lambda_{\text {err }}$ ), dimensionless

$B i_{h}$ Biot number based on particle diameter for the cold inner tube in the large set-up $\left(=\alpha_{w, h} d_{p} / \lambda_{\text {eff }}\right)$, dimensionless

$B i_{r}^{\ominus}$ modified Biot number at the cold wall ( $\left.=\alpha_{w, c} R_{u} / \lambda_{\text {eff }}\right)$, dimensionless

$B i_{h}^{\ominus}$ modified Biot number at the hot wall $\left(=\alpha_{w, h} R_{w} / \lambda_{\text {eff }}\right)$, dimensionless

$C_{p, g} \quad$ specific heat of the gas, $J / \mathrm{kg} \mathrm{K}$

$C_{p, k} \quad$ specific heat of the solid, $J / \mathrm{kg} \mathrm{K}$

$d_{p}$ particle diameter equivalent to a sphere $\left(=6 V_{p} / A_{p}\right), \mathrm{m}$

$I_{0} \quad$ zeroth-order modified Bessel function of the first kind

$J_{0}, J_{1}$ zeroth- and first-order Bessel functions of the first kind

$L_{\text {v }}$ tube length, length of the packed bed, $\mathrm{m}$

$N_{D} \quad$ number of particies on a diameter $\left(=2 R_{w} / d_{p}\right)$, dimensionless

$N u \quad$ particle Nusselt number $\left(=\alpha_{p} d_{p} / \lambda_{g}\right)$, dimensionless

Pe particle Peclet number $\left(=v_{0} d_{p} / a_{g}\right)$, dimensionless

$P e^{\ominus}$ modified Peclet number based on tube dimensions $\left[=\rho_{g} C_{p, g} v_{0} R_{u}^{2} /\left(\lambda_{\text {ef }} L_{t}\right)\right]$, dimensionless

$r \quad$ radial coordinate, $m$

$\boldsymbol{R}_{i} \quad$ inner radius packed bed, $\mathrm{m}$

$\boldsymbol{R}_{\boldsymbol{u}} \quad$ outer radius packed bed, $m$

$S t^{\ominus} \quad$ modified Stanton number $\left[=6(1-\varepsilon) \alpha_{p} L_{t} /\right.$ $\left.\left(\rho_{p} C_{p \cdot \theta} v_{0} d_{p}\right)\right]$, dimensionless

$t$ time, $s$

$T_{b} \quad$ initial temperature of the packed bed, $\mathrm{K}$

$T_{c} \quad$ temperature of the cold inner tube, $K$

$T_{\theta} \quad$ gas temperature, $\mathbf{K}$
$T_{h} \quad$ temperature of the hot outer wall, $\mathrm{K}$

$T_{i \text { in }} \quad$ gas inlet temperature, $\mathbf{K}$

$T_{k} \quad$ pellet temperature, $\mathrm{K}$

$v_{0} \quad$ superficial gas velocity based on the empty tube, $\mathrm{m} / \mathrm{s}$

$V$ volume of a pellet, $\mathrm{m}^{3}$

$Y_{0}, Y_{1}$ zeroth- and first-order Bessel functions of the second kind

$z \quad$ axial coordinate, $m$

\section{Greek letters}

$\alpha_{p} \quad$ heat transfer coefficient between the particle and the gas, $W / m^{2} K$

$\alpha_{w} \quad$ heat transfer coefficient between the packed bed and the wall, $\mathrm{W} / \mathrm{m}^{2} \mathrm{~K}$

$\boldsymbol{\alpha}_{w, c} \quad$ heat transfer coefficient at the wall of the cold inner tube, $W / \mathbf{m}^{2} K$

$\alpha_{w, h} \quad$ heat transfer coefficient at the wall of the hot outer wall, $\mathrm{W} / \mathrm{m}^{2} \mathrm{~K}$

$\Gamma \quad$ dimensionless effective radial heat conductivity $\left(=\lambda_{\text {err }} / \lambda_{g}\right)$

$\varepsilon$ porosity, volume fraction of the gas in the bed, dimensionless

$\theta_{b}$ dimensionless initial temperature of the bed $\left[=\left(T_{b}-T_{c}\right) /\left(T_{h}-T_{c}\right)\right]$

$\theta_{g}$ dimensionless gas temperature $\left[=\left(T_{g}-T_{c}\right) /\right.$ $\left.\left(T_{h}-T_{c}\right)\right]$

$\theta_{\text {in }}$ dimensionless gas inlet temperature $\left[=\left(T_{\text {in }}-T_{c}\right) /\left(T_{h}-T_{c}\right)\right]$

$\theta_{k}$ dimensionless pellet temperature $\left[=\left(T_{k}-\right.\right.$ $\left.\left.T_{c}\right) /\left(T_{h}-T_{c}\right)\right]$

ratio of inner to outer radius of the packed bed ( $=\boldsymbol{R}_{i} / \boldsymbol{R}_{\mathrm{u}}$ ), dimensionless

$\lambda_{\text {eff }}$ effective radial heat conductivity of the packed bed, $\mathbf{W} / \mathrm{m} \mathrm{K}$

$\lambda_{g}$ heat conductivity of the gas, $W / m ~ K$

$\Lambda$ tube slenderness $\left[=L_{v} /\left(2 R_{w}\right)\right]$, dimensionless

$\rho$ dimensionless radial coordinate $\left(=r / R_{\mu}\right)$

$\rho_{0}$ density of the gas, $\mathrm{kg} / \mathrm{m}^{3}$

$\rho_{k}$ density of the pellet, $\mathrm{kg} / \mathrm{m}^{3}$

$\tau$ dimensionless time $\left\{=\left(v_{0} t / L\right) \times\left[\varepsilon \rho_{g} C_{p, g}\right) /\right.$ $\left.\left\lceil(1-\varepsilon) \rho_{k} C_{p, k}\right]\right\}$

$\omega$

\section{REFERENCES}

Bauer, R., 1977, Effective radial thermal conductivity of gas-permeated packed beds containing particles of different shape and size distribution. VDI Forschungsh. 582, 39.

Cresswell, D. L., 1986, Heat transfer in packed beds. NATO ASI Ser., Ser. E, Chem. Reac. Des. Technol. 110, 687-728.

Dixon, A. G. and Cresswell, D. L., 1979, Theoretical prediction of effective heat transport parameters in packed beds. A.I.Ch.E. J. 25(4), 663-676.

Gnielinski, V., 1980, Heat and mass transfer in packed beds. Chem. Ing. Technik 52(3), 228-236.

Gnielinski, V., 1982, Berechnung des Wärme- und Stoffaustausch in durchströmten ruhenden Schüttungen. Verfahrenstechnik 16, 36-39.

Gunn, D. J. and de Souza, J. F. C., 1974, Heat transfer and axial dispersion in packed beds. Chem. Engng Sci. 29, 1363-1371.

Hennecke, F. W. and Schlünder, E. U., 1973, Heat transfer in 
heated or cooled tubes with packings of spheres, cylinders and Raschig rings. Chem. Ing. Technik 4S(5), 277-284.

Hofmann, H., 1979, Fortschritte bei der Modellierung von Festbettreaktoren. Chem. Ing. Technik 51(4), 257-265.

Kunii, D. and Suzuki, M., 1967, Particle-to-fluid heat and mass transfer in packed beds of fine particles. Int. J. Heat Mass Transfer 10, 845-852.

Lerou, J. J. and Froment, G. F., 1978, Estimation of heat transfer parameters in packed beds from radial temperature profiles. Chem. Engng J. (Lausanne) 15(3), 233-237.

Martin, H., 1978, Low Peclet number particle-to-fluid heat and mass transfer in packed beds. Chem. Engng Sci. 33, 913-919.

Nelson, P. A. and Galloway, T. R., 1975, Particle-to-fluid heat and mass transfer in dense systems of fine particles. Chem. Engng Sci. 30, 1-6.

Ranz, W. E., 1952, Friction and transfer coefficients for single particles and packed beds. Chem. Engng Prog. 48, 247-253.

Tsutsas, E. and Martin, $H_{\text {., }}$ 1987, Thermal conductivity of packed beds: a review. Chem. Engng Process. 22, 19-37.

Vortmeyer, D. and Haidegger, E., 1991, Discrimination of three approaches to evaluate heat fluxes for wall-cooled fixed bed chemical reactors. Chem. Engng Sci. 46, 2651-2660.

Wellauer, T., Cresswell, D. L. and Newson, E. L., 1982, Heat Iransfer in packed bed reactor tubes suitable for selective oxidation. ACS Symp. Ser. 169, 527-543.

Westerterp, K. R., van Swaaij, W. P. M. and Beenackers, A. A. C. M., 1987, Chemical Reactor Design and Operation. Wiley, Chichester.

Wijngaarden, R. J., 1988, The scaling up of cooled tubular reactors. Thesis, Twente University, Enschede.

Wijngaarden, R. J. and Westerterp, K. R., 1992, The statistical character of packed-bed heat transport properties. Chem. Engng Sci. 47, 3125-3129.

Zehner, P., 1973, Experimental and theoretical determination of the effective heat conductivity of solid beds consisting of spheres with throughflow at moderate and high temperatures. VDI Forschungsh. 558, 35.

Zehner, P. and Schlünder, E. U., 1973, Effective thermal conductivity of spherical packings perfused at moderate and high temperatures. Chem. Ing. Technik 45(5), 272-276.

\section{APPENDIX}

The set of partial differential equations (1) and (2) subject to the initial conditions (3) and (4) and the boundary conditions (5) and (6) was solved by taking the Laplace transform of this set with respect to $\tau$ and $\omega$. Inversion from the Laplace domain was done with the aid of the complex inversion formula.

The result is

$$
\begin{aligned}
& \theta_{a}=\frac{1 / t-B i_{c}^{\Theta} \ln (\rho / \imath)}{1 / \imath-B i_{c}^{\Theta} \ln t+\left(B i_{c}^{\Theta} / B i_{h}^{\Theta}\right)}+\sum_{i=1}^{\infty}\left\langle\frac { 2 } { \lambda _ { i } } \left\{\left[\frac{\Theta\left(\lambda_{i}\right)-\theta_{b} \Xi\left(\lambda_{i}\right)}{\Phi\left(\lambda_{i}\right)}\right]\right.\right. \\
& \times\left(\frac{1}{1+\eta_{i}}\right)\left\{\left[1-\frac{e^{-\left(1+\eta_{i}\right)}}{1+\eta_{i}}\right.\right. \\
& \times \int_{0}^{S \theta^{\theta_{\tau}}} I_{0}\left(2 \sqrt{\left.S t^{\ominus} \omega u\right)} e^{-\psi /\left(1+\eta_{1}\right)} \mathrm{d} u\right] \\
& x e^{-n_{i} S t^{\theta} \tau /\left(1+n_{i}\right)}+e^{-S t^{\theta_{\tau}} \tau-\left(1+n_{i} S t^{\circ} \omega\right.}
\end{aligned}
$$

$$
\begin{aligned}
& \times I_{0}\left(2 S t^{\Theta} \sqrt{\omega \tau)}\right\}+\left[\frac{\Theta\left(\lambda_{i}\right)-\theta_{0} \Xi\left(\lambda_{i}\right)}{\Phi\left(\lambda_{i}\right)}\right]\left[e^{-\left(1+\eta_{i} S t^{\theta} \omega\right)}\right.
\end{aligned}
$$

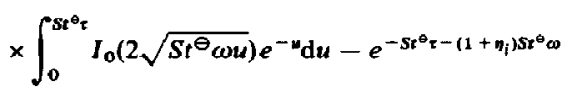

$$
\begin{aligned}
& \left.\left.\left.\times I_{0}\left(2 S t^{\ominus} \sqrt{\omega \tau}\right)\right]\right\}\right\rangle \\
& \theta_{k}=\frac{1 / t-B i_{c}^{\Theta} \ln (\rho / t)}{1 / t-B i_{c}^{\Theta} \ln t+\left(B i_{c}^{\Theta} / B i_{h}^{\Theta}\right)}+e^{-S t^{\theta_{c}}} \\
& \times\left[\theta_{b}-\frac{1+B i_{c}^{\ominus} \ln (\rho / l)}{1 / l-\ln \iota+\left(B i_{c}^{\ominus} / B i_{h}^{\ominus}\right)}\right] \\
& +\sum_{i=1}^{\infty}\left(\frac { 2 } { \lambda _ { i } } \left\langle\left[\frac{\Theta\left(\lambda_{i}\right)-\theta_{b} \Xi\left(\lambda_{i}\right)}{\Phi\left(\lambda_{i}\right)}\right] \times\left\{e^{-\eta_{i} S \theta^{\theta} \tau /\left(1+n_{i}\right)}\right.\right.\right. \\
& \left.\times\left[1-\frac{e^{-\left(1+\eta_{i}\right)}}{1+\eta_{i}} \int_{0}^{S t^{\theta_{\tau}}} I_{0}\left(2 \sqrt{S t^{\theta} \omega u}\right) e^{-u /\left(1+\eta_{i}\right)} \mathrm{d} u\right]-e^{-s t^{-\theta_{i}}}\right\} \\
& +\left[\frac{\Theta\left(\lambda_{i}\right)-\theta_{0} \Xi\left(\lambda_{i}\right)}{\Phi\left(\lambda_{i}\right)}\right] e^{-\left(1+\eta_{i} s i^{\Theta} \omega\right)} \\
& \left.\left.\times \int_{0}^{S t^{e_{x}}} I_{0}\left(2 \sqrt{S t^{\ominus} \omega u}\right) e^{-\| \mathrm{d} u}\right\rangle\right)
\end{aligned}
$$

with

$$
\begin{aligned}
& \eta_{i}=\frac{\lambda_{i}^{2}}{P e^{\theta} S t^{\theta}} \\
& \Theta(\lambda)=P_{0}(\lambda \rho, \lambda z)-\frac{\lambda}{B i_{c}^{\theta}} Q_{0}(\lambda \rho, \lambda l) \\
& \Xi(\lambda)=P_{0}(\lambda, \lambda \rho)+P_{0}(\lambda \rho, \lambda l)-\frac{\lambda}{B i_{c}^{G}} Q_{0}(\lambda \rho, \lambda z) \\
& -\frac{\lambda}{B i_{h}^{\ominus}} Q_{0}(\lambda \rho, \lambda) \\
& \Phi(\lambda)=\lambda P_{0}(\lambda, \lambda)\left(\frac{l}{B i_{c}^{\Theta}}-\frac{1}{B i_{h}^{\theta}}\right)+\lambda P_{1}\left(\lambda, \lambda_{l}\right)\left(\frac{t}{B i_{h}^{\ominus}}-\frac{1}{B i_{c}^{\theta}}\right) \\
& +Q_{0}(\lambda, \lambda l)\left(l+\frac{\lambda^{2}}{B i_{c}^{\ominus} B i_{h}^{\ominus}}\right)-Q_{0}(\lambda l, \lambda)\left(1+\frac{\lambda^{2} l}{B i_{c}^{\Theta} B i_{h}^{\ominus}}\right)
\end{aligned}
$$

and $\lambda_{i}$ the ith root of the equation

$$
\begin{gathered}
P_{0}(\lambda, \lambda l)-\frac{\lambda}{B i_{h}^{\Theta}} Q_{0}(\lambda l, \lambda)-\frac{\lambda}{B i_{c}^{\Theta}} Q_{0}(\lambda, \lambda l) \\
-\frac{\lambda^{2}}{B i_{c}^{\Theta} B i_{h}^{\Theta}} P_{1}(\lambda, \lambda l)=0 .
\end{gathered}
$$

$P_{0}, P_{1}$ and $Q_{0}$ are the following cross-products:

$$
\begin{aligned}
& P_{0}(a, b)=J_{0}(a) Y_{0}(b) \quad Y_{0}(a) J_{0}(b) \\
& P_{1}(a, b)=J_{1}(a) Y_{1}(b)-Y_{1}(a) J_{1}(b) \\
& Q_{0}(a, b)=-J_{0}(a) Y_{1}(b)+Y_{0}(a) J_{1}(b) .
\end{aligned}
$$

For symbols see the Notation. 